\title{
U-Omp19 from Brucella abortus Is a Useful Adjuvant for Vaccine Formulations against Salmonella Infection in Mice
}

\author{
Gabriela S. Risso', Marianela V. Carabajal', Laura A. Bruno', Andrés E. Ibañez', \\ Lorena M. Coria', Karina A. Pasquevich', Seung-Joo Lee', Stephen J. McSorley², \\ Gabriel Briones' ${ }^{1}$ and Juliana Cassataro ${ }^{1 *}$

\begin{abstract}
"Instituto de Investigaciones Biotecnológicas "Rodolfo Ugalde"-Instituto Tecnológico de Chascomús (IIB-INTECH), Universidad Nacional de San Martín (UNSAM), Consejo Nacional de Investigaciones Científicas y Técnicas (CONICET), Buenos Aires, Argentina, ${ }^{2}$ Center for Comparative Medicine (CCM), Department of Anatomy, Physiology and Cell Biology, School of Veterinary Medicine, University of California Davis, Davis, CA, USA
\end{abstract}

OPEN ACCESS

Edited by:

Rashika El Ridi,

Cairo University, Egypt

Reviewed by:

Renato De Lima Santos,

Universidade Federal de Minas

Gerais, Brazil

Yasmin Thanavala,

Roswell Park Cancer Institute, USA

Caroline Junqueira,

Oswaldo Cruz Foundation, Brazil

${ }^{*}$ Correspondence:

Juliana Cassataro

jucassataro@iibintech.com.ar

Specialty section: This article was submitted to Vaccines and Molecular Therapeutics,

a section of the journal

Frontiers in Immunology

Received: 31 October 2016 Accepted: 03 February 2017 Published: 17 February 2017

Citation:

Risso GS, Carabajal MV, Bruno LA, Ibañez $A E$, Coria $L M$, Pasquevich KA, Lee S-J, McSorley SJ, Briones G and Cassataro J (2017) U-Omp19 from

Brucella abortus Is a Useful Adjuvant for Vaccine Formulations against Salmonella Infection in Mice.

Front. Immunol. 8:171. doi: 10.3389/fimmu.2017.00171
Most pathogens infect through mucosal surfaces, and parenteral immunization typically fails to induce effective immune responses at these sites. Development of oral-administered vaccines capable of inducing mucosal as well as systemic immunity while bypassing the issues of antigen degradation and immune tolerance could be crucial for the control of enteropathogens. This study demonstrates that U-Omp19, a bacterial protease inhibitor with immunostimulatory features, coadministered with Salmonella antigens by the oral route, enhances mucosal and systemic immune responses in mice. U-Omp19 was able to increase antigen-specific production of IFN- $\gamma$ and IL-17 and mucosal (IgA) antibody response. Finally, oral vaccination with U-Omp19 plus Salmonella antigens conferred protection against virulent challenge with Salmonella Typhimurium, with a significant reduction in bacterial loads. These findings prove the efficacy of this novel adjuvant in the Salmonella infection model and support the potential of U-Omp19 as a suitable adjuvant in oral vaccine formulations against mucosal pathogens requiring Thelper (Th)1-Th17 protective immune responses.

Keywords: adjuvant, vaccination, Salmonella Typhimurium, T helper 1, T helper 17

\section{INTRODUCTION}

It is generally accepted that vaccination is the most efficient and cost-effective form of preventing infectious diseases. Although most vaccines currently licensed are administered by parenteral route, this vaccination strategy usually fails to elicit adequate mucosal immune responses. As a result, diseases caused by mucosal pathogens are still among the major causes of death in developing countries. It is well documented that oral immunization is capable of generating strong protective immunity at the intestinal mucosa as well as systemically $(1-5)$. However, oral administration of antigens faces two major issues, one being degradation of the antigen by enzymes present at the gastrointestinal tract and the other being the induction of immune tolerance against the administered antigen (6-8). Current oral vaccines based on attenuated pathogens are usually capable of bypassing these difficulties but, on the other hand, present important safety concerns (9). The safe alternative consists on the development of oral killed or subunit vaccines, but this holds major challenges since they are poorly 
immunogenic and usually require multiple doses and the use of an effective oral adjuvant $(10,11)$.

Two bacterial products are being used as oral adjuvants in the mouse model, cholera toxin (CT) from Vibrio cholerae and heatlabile enterotoxin (LT) from Escherichia coli (12). Since enterotoxicity seriously limits the practical use of these compounds in humans, modifications have been generated to reduce this effect. A modified version of CT lacking the A subunit (CTB) is now currently licensed as part of the Dukoral ${ }^{\circledR}$ vaccine for human use (13) and also a double mutant of LT, which retains its adjuvant properties is under clinical trial (14). However, neither of these molecules is capable of overcoming antigen degradation in the gastrointestinal tract. Interesting strategies to address this issue include antigen delivery through intestine-targeted $\mathrm{pH}$-dependent microparticles (15), biodegradable nano or microparticles (16-18), and antigen targeting to M cells (19). However, there is still a need for an appropriate oral adjuvant, especially one that induces $\mathrm{T}$ helper (Th) 1 and $\mathrm{CD}^{+} \mathrm{T}$ cell immune responses required for protective immunity against intracellular pathogens.

In previous work we have studied the unlipidated form of a bacterial protease inhibitor from Brucella abortus, U-Omp19, as an oral adjuvant. We have demonstrated that it has selfadjuvanting properties (20) and can also enhance immune responses against the model antigen chicken ovalbumin (OVA) $(21,22)$. The mechanisms responsible for the adjuvant activity of $\mathrm{U}$-Omp19 rely in the inhibition of stomach and gut proteases and recruitment of immune cells to the gastrointestinal mucosa (21) as well as increased maturation of DCs and enhanced presentation of the antigen by means of delaying antigen digestion at the lysosomes (23). Thus, mucosal as well as systemic OVA-specific immune responses, Th1, and $\mathrm{CD}^{+}$are enhanced if $\mathrm{U}-\mathrm{Omp} 19$ is codelivered orally.

We now focused on assessing U-Omp19's capacity to increase immune response against antigens from an enteric pathogen that invades the host through the gastrointestinal mucosa. The enterobacteria Salmonella infect humans and animals and depending on the serotype can cause disease of different severity (24). S. Typhi invades the gut mucosa and is drained trough the lymphatic system into lymph nodes, spleen, and liver (25). The main structural protein of Salmonella flagellum is Flagellin (FliC). Flagellin was demonstrated to play an important role in the protection against Salmonella challenge (26). Also, a significant fraction of Salmonella-specific $\mathrm{CD} 4^{+} \mathrm{T}$ cells respond to FliC, and this antigen has the capacity to protect naive mice from lethal Salmonella infection (27). Another protein expressed and secreted by Salmonella is SseB, which promotes membrane pore formation, allowing proteins to access host cytoplasm. SseB was highlighted in a proteomic screen and has been shown to protect mice against Salmonella infection (28). Th1 immunity involving IFN- $\gamma$ is strongly associated with the protective immune response to Salmonella (29-31). The natural route of infection and the need for a Th1-biased response, along with the current emergence of multidrug-resistant strains, makes this pathogen a strong candidate for oral vaccination with the novel adjuvant U-Omp19. The conventional mouse model for the study of this disease is infection of susceptible mouse strains with Salmonella enterica serovar Typhimurium (S. Typhimurium) that causes an invasive systemic disease that is similar in many respects to typhoid fever (30-32). Therefore, in this work U-Omp19 was studied in vivo for its ability to elicit mucosal and systemic immunity against coadministered antigens from $S$. Typhimurium. We assessed a killed-vaccine immunization alternative, using a heat-killed extract of $S$. Typhimurium (HKS) as well as a proof of concept using FliC and SseB antigens in a potential subunit vaccine alternative.

\section{MATERIALS AND METHODS}

\section{Ethics Statement}

All experimental protocols of this study were conducted in agreement with international ethical standards for animal experimentation (Helsinki Declaration and its amendments, Amsterdam Protocol of welfare, and animal protection and National Institutes of Health, USA, guidelines: Guide for the Care and Use of Laboratory Animals). The protocols of this study were approved by the Institutional Committee for the Care and Use of Experimentation Animals from the University of San Martin (UNSAM) or from University of California Davis.

\section{Mice}

BALB/c and CF-1 mice were obtained from Animal Facility at IIB-UNSAM or from Jackson Laboratory and were used at 6-12 weeks of age. Mice were housed in appropriate conventional animal care facilities and handled according to international guidelines required for animal experiments at IIB-UNSAM or at University of California Davis.

\section{Antigens and Adjuvants}

The recombinant unlipidated (U-) Omp19 was expressed and purified as previously described (33). Lipopolysaccharide (LPS) contamination was adsorbed with Sepharose-polymyxin B (SIGMA, St. Louis, MO, USA). Endotoxin and protein concentration were determined as in Ref. (21). All U-Omp19 preparations used contained $<0.10$ endotoxin $\mathrm{U} / \mathrm{mg}$ of protein. CT and CT subunit B (CTB) from $V$. cholerae were purchased from SIGMA, St. Louis, MO, USA.

For preparation of heat-killed extract of Salmonella Typhimurium (HKS), AroA S. Typhimurium (streptomycin resistant) was plated in LB agar with $10 \mu \mathrm{g} / \mathrm{ml}$ of streptomycin and grown overnight at $37^{\circ} \mathrm{C}$. Afterward, eight isolated colonies were taken from the LB plate and grown in LB medium with $10 \mu \mathrm{g} /$ $\mathrm{ml}$ of streptomycin at $37^{\circ} \mathrm{C}$ with agitation of $130 \mathrm{rpm}$ overnight. The next morning, the culture was diluted 1 in 25 in LB medium with $10 \mu \mathrm{g} / \mathrm{ml}$ of streptomycin and grown until reaching an OD $600 \mathrm{~nm}$ of $0.85\left(\approx 1 \times 10^{9} \mathrm{CFU} / \mathrm{ml}\right)$, for the bacterial culture to be in growth phase. After centrifugation for $10 \mathrm{~min}$ at $6,000 \mathrm{rpm}$ at $4^{\circ} \mathrm{C}$, supernatant was discarded and bacterial pellet was suspended in sterile PBS. This bacterial extract was then heated at $63^{\circ} \mathrm{C}$ for $3 \mathrm{~h}$; afterward it was heated at $100^{\circ} \mathrm{C}$ for $10 \mathrm{~min}$ and finally sonicated for $10 \mathrm{~min}$ in an ice-cold environment. Protein concentration was determined by the bicinchroninic acid assay (Pierce, Rockford, IL, USA) and aliquots of $1-5 \times 10^{6} \mathrm{CFU} / \mu \mathrm{g}$ of extract were stored at $-70^{\circ} \mathrm{C}$ until used. 
SseB was obtained as described in Ref. (28). Flagellin was purified from LPS-deficient $S$. Typhimurium X4700 as in Ref. (34).

\section{Antigen Degradation Studies}

To obtain the mouse stomach extract, five mice were fasted overnight. Stomach was obtained from every mouse and mechanically homogenized in $500 \mu \mathrm{l}$ of ice-cold PBS (Ultra-Turrax homogenizer, IKA). Homogenates were pooled, immediately centrifuged $15 \mathrm{~min}$ at $6,000 \mathrm{rpm}$, and supernatants were used as mouse stomach extract. Standardization of extracts was done by measuring (i) total protein content by the bicinchroninic acid assay (Pierce, Rockford, IL, USA) and (ii) protease activity of the extract. Protease activity was determined using a casein fluorimetric kit (EnzChek, Invitrogen, Carlsbad, CA, USA). EnzChek kit contains casein BODIPY FL, a protein in which fluorescence is quenched. Protease-catalyzed hydrolysis releases this quenching, yielding bright green fluorescent peptides. The increase in fluorescence is proportional to protease activity.

To examine whether U-Omp19 would limit digestion of HKS by stomach proteases, HKS was incubated with stomach extracts with or without U-Omp19 for $5 \mathrm{~h}$. Following incubation, each mixture of reaction was separated on $12 \%$ SDS-PAGE. To increase sensitivity of this assay we performed Western Blot against FliC (Salmonella Flagellum Protein-FliC-) an antigen that has shown to be protective in mice $(26,27)$. Membrane was incubated overnight with anti-FliC IgG MAb (Invivogen Carlsbad, CA, USA), followed by 1-h incubation with anti-IgG-HRP (SIGMA, St. Louis, MO, USA), and reveled using ECL kit (Pierce, Rockford, IL, USA). This helped us determine if U-Omp19 can protect digestion of this particular protein in the heat-killed preparation of Salmonella.

\section{Immunization}

$\mathrm{BALB} / \mathrm{c}$ and CF-1 mice were immunized orally-intragastrically by oral gavage-with HKS (100 $\left.\mu \mathrm{g} \approx 1-5 \times 10^{8} \mathrm{CFU}\right)$ alone, with U-Omp19 $(200 \mu \mathrm{g})$, with CTB or with CT $(10 \mu \mathrm{g})$ on days 0 and 7. These were diluted in PBS until a final volume of $200 \mu \mathrm{l}$ per administration per mouse. Dose of U-Omp19 was chosen considering previous studies on protease inhibitory capacity of U-Omp19 in vivo (21), whereas CT dose was selected for being the typical effective oral dose described in the literature (35-38). Mice were fasted $2 \mathrm{~h}$ before and after immunization. Fifteen minutes before oral immunization, mice were administered with $100 \mu \mathrm{l}$ of $0.1 \mathrm{M}$ sodium bicarbonate.

For subunit vaccine experiments, BALB/c mice were intravenously or orally immunized on days 0 and 30 with (i) buffer, (ii) $20 \mu \mathrm{g} \mathrm{SseB}+20 \mu \mathrm{g}$ Flagellin $+80 \mu \mathrm{g}$ U-Omp19, or (iii) $20 \mu \mathrm{g}$ SseB $+20 \mu$ F Flagellin + $10 \mu \mathrm{g}$ monophosphoryl lipid A (MPLA) (Invivogen, Carlsbad, CA, USA) as a control adjuvant.

\section{Determination of Th Immune Responses}

Spleen and MLNs' single cell suspensions from immunized mice were obtained as in Ref. (21) 14 days post-last immunization and cells' suspensions were cultured in the presence of 0.5 or 10 micrograms $/ \mathrm{ml}$ of HKS or complete medium. After 3 days, cell culture supernatants were collected and IFN- $\gamma$, IL-4, IL-10, and IL-17 were determined by ELISA (Pharmingen, San Diego, CA, USA).

\section{Delayed Type Hypersensitivity Assay (DTH) Test}

Two weeks after the last immunization, mice were injected intradermally in one footpad with $20 \mu \mathrm{g}$ of HKS in $40 \mu \mathrm{l}$ of saline and in the contralateral footpad with an equal volume of saline. Footpad thickness was measured $72 \mathrm{~h}$ later using a digital caliper with a precision of $0.01 \mathrm{~mm}$, and the mean increase in footpad thickness (mm) was calculated as (footpad thickness) HKS - (footpad thickness) saline.

\section{Determination of IgA in Feces and IgG in Sera}

Two weeks after the last immunization, fecal extracts were prepared as in Ref. (20) and used on the same day. Mice were bled via the cheek pouch on the submandibular vein. Blood was allowed to clot, and serum was removed and stored at $-20^{\circ} \mathrm{C}$ until used.

For ELISA, HKS extract was diluted to $1 \mathrm{mg} / \mathrm{ml}$ in $\mathrm{NaHCO}_{3} 1 \mathrm{M}$ $\mathrm{pH}$ 9.6, and ELISA plates were coated with $100 \mu \mathrm{g} /$ well overnight at $4^{\circ} \mathrm{C}$. Plates were blocked with bovine serum albumin $2 \%$ in PBS at $37^{\circ} \mathrm{C}$ for $1 \mathrm{~h}$ and washed with PBS-Tween $0.05 \%$. Samples were incubated for $2 \mathrm{~h}$ at room temperature and after washing, antimouse IgA-HRP (Abcam, Cambridge, MA, USA) or anti-mouse IgG-HRP (SIGMA, St. Louis, MO, USA) diluted in PBS-BSA 1\% were added for $1 \mathrm{~h}$ at room temperature. Finally, detection was performed with BD OptEIA ${ }^{\mathrm{TM}}$ TMB Substrate Reagent Set (BD, San Diego, CA, USA). Anti-U-Omp19 ELISA was performed as previously described (33).

\section{Protection Experiments HKS Experiments}

For BALB/c mice, 14 days post-last immunization, mice from each group were challenged intragastrically with $0.5-1 \times 10^{5} \mathrm{CFU}$ of virulent $S$. Typhimurium. For CF- 1 mice, 21 days post-last immunization, mice from each group were challenged intragastrically with $1-5 \times 10^{5} \mathrm{CFU}$ of virulent $S$. Typhimurium. Mice were fasted for $6 \mathrm{~h}$ before and $1 \mathrm{~h}$ after infection. Fifteen minutes before infection mice were administered intragastrically with $100 \mu \mathrm{l}$ of $\mathrm{NaHCO}_{3} 0.1 \mathrm{M}$ pH8. Sacrifice was performed 6 days postinfection for $\mathrm{BALB} / \mathrm{c}$ mice and 40 days postinfection for CF- 1 mice. Spleens and livers were obtained and homogenized in sterile PBS. Serial dilutions of the homogenates were plated on SS agar plates and incubated overnight at $37^{\circ} \mathrm{C}$ to determine bacterial colonization.

\section{Recombinant Antigens Experiments}

Thirty days post-last immunization, mice were intravenously (tail vein) infected with 1,000 CFU of virulent Salmonella SL1344 strain. Four days later spleen and liver were obtained, and bacterial load was determined by homogenization, serial dilution, and plating on MacConkey agar plates.

\section{Statistical Analysis}

Statistical analysis and plotting were performed using GraphPad Prism 5 software. Data (with logarithmic transformation when 
necessary) were tested for normality and homoscedasticity before using parametric statistics (one-way ANOVA) or analyzed using non-parametric statistics (Kruskal-Wallis). Data were tested for normality using the Kolmogorov-Smirnov test, and for equal variance, using the Levene Median test. Results shown are representative of at least two independent experiments. Results were expressed as mean \pm SEM. Significance level was set at $p<0.05$.

\section{RESULTS}

\section{U-Omp19 Protects HKS Antigens from Stomach Digestion}

Previous studies have shown that U-Omp19 has inhibitory activity over several proteases at the digestive tract, particularly pepsin, the main protease present at the stomach (21). Thus, U-Omp19's activity in an in vitro degradation assay incubating HKS with mouse stomach extract was evaluated. One of the antigens present in the HKS extract is FliC. FliC is a major protein and virulence factor from Salmonella that has the capacity to protect naive mice from lethal Salmonella infection (26-28), so if degradation of FliC by stomach enzymes is reduced, more FliC can reach inductive sites and thus it is likely to be able to perform as a protective antigen in an oral vaccine. Detection of FliC by western blot confirms that U-Omp19 protects antigen from degradation by stomach enzymes in vitro (Figure S1 in Supplementary Material), which may contribute to the induction of a stronger immune response.

\section{Vaccination with HKS Plus U-Omp19 Induces an Increase in Mucosal Antibody Response}

There is evidence that antibodies are associated with protection against $S$. Typhimurium $(39,40)$. To assess U-Omp19's adjuvant effect on the antibody response against HKS antigens, HKS-specific antibody production in orally immunized mice was examined. Intestinal HKS-specific IgA antibodies were significantly elevated in fecal pellets from mice immunized with HKS plus U-Omp19 compared to those immunized with HKS alone. Serum HKS-specific IgG levels, on the other hand, were not altered if U-Omp19 was coadministered with HKS. Of note, immunization with $\mathrm{U}-\mathrm{Omp} 19$ as adjuvant did not induce a mucosal antibody response against itself (Figure 1).

\section{U-Omp19 Induces Systemic and Mucosal Cellular Immune Responses against Coadministered HKS Antigens}

Since the cellular immune response has been reported to be important for protection against Salmonella (41-43), the capacity of U-Omp19 to increase HKS-specific cell-mediated immune response in a DTH assay was tested in immunized mice. After $72 \mathrm{~h}$ of intradermal administration of HKS, mice that had been previously delivered with HKS + U-Omp19 displayed an increased DTH response compared to the HKS-immunized group (Figure 2A). The profile of cytokine production upon antigen stimulation was studied after intragastric immunization with HKS, HKS + U-Omp19, or HKS + CT as mucosal adjuvant control. Splenocytes from HKS + U-Omp19 immunized mice presented a significant increase in IFN- $\gamma$ and IL-17 production upon HKS stimulation in vitro in comparison to HKS alone immunization. In contrast, HKS stimulation in vitro did not increase IL-4 or IL-10 production (Figure 2B). CT induced a statistically significant increase in IL-17 and a slight but not significant increase in IFN- $\gamma$.

To evaluate U-Omp19's effect on HKS-specific mucosal immune response, MLNs from immunized mice were obtained and stimulated in vitro with HKS. As seen in splenocytes, IFN- $\gamma$ and IL-17 levels in supernatants from MLNs' cells were increased in animals immunized with HKS + U-Omp19 compared to HKS alone (Figure 2C). Production of IL-4 and IL-10 did not differ between groups (data not shown).

\section{Coadministration of HKS with U-Omp19 Reduces Bacterial Burden after Oral Challenge with $S$. Typhimurium}

To evaluate if the induction of an adaptive immune response by HKS + U-Omp19 would lead to an increased level of protection against infection, immunized mice were orally infected with
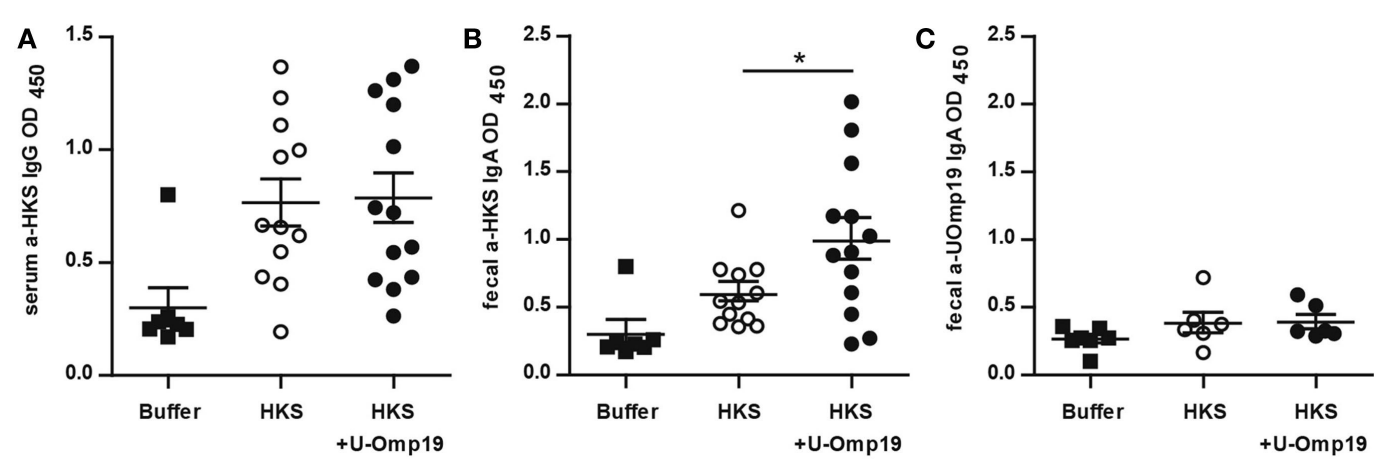

FIGURE 1 | U-Omp19 induces an increase in fecal IgA antibodies against HKS. BALB/c mice were orally immunized on days 0 and 7 with buffer, HKS alone, or HKS + U-Omp19. Fourteen days post-last immunization, sera and feces were collected. HKS-specific IgG in serum (A), HKS-specific IgA in fecal extracts (B), and U-Omp19-specific IgA in fecal extracts $\mathbf{( C )}$ were detected by ELISA. Individual mice are shown as scatter dots ( ${ }^{*} p<0.05$; ANOVA plus Bonferroni's multiple comparisons test). 

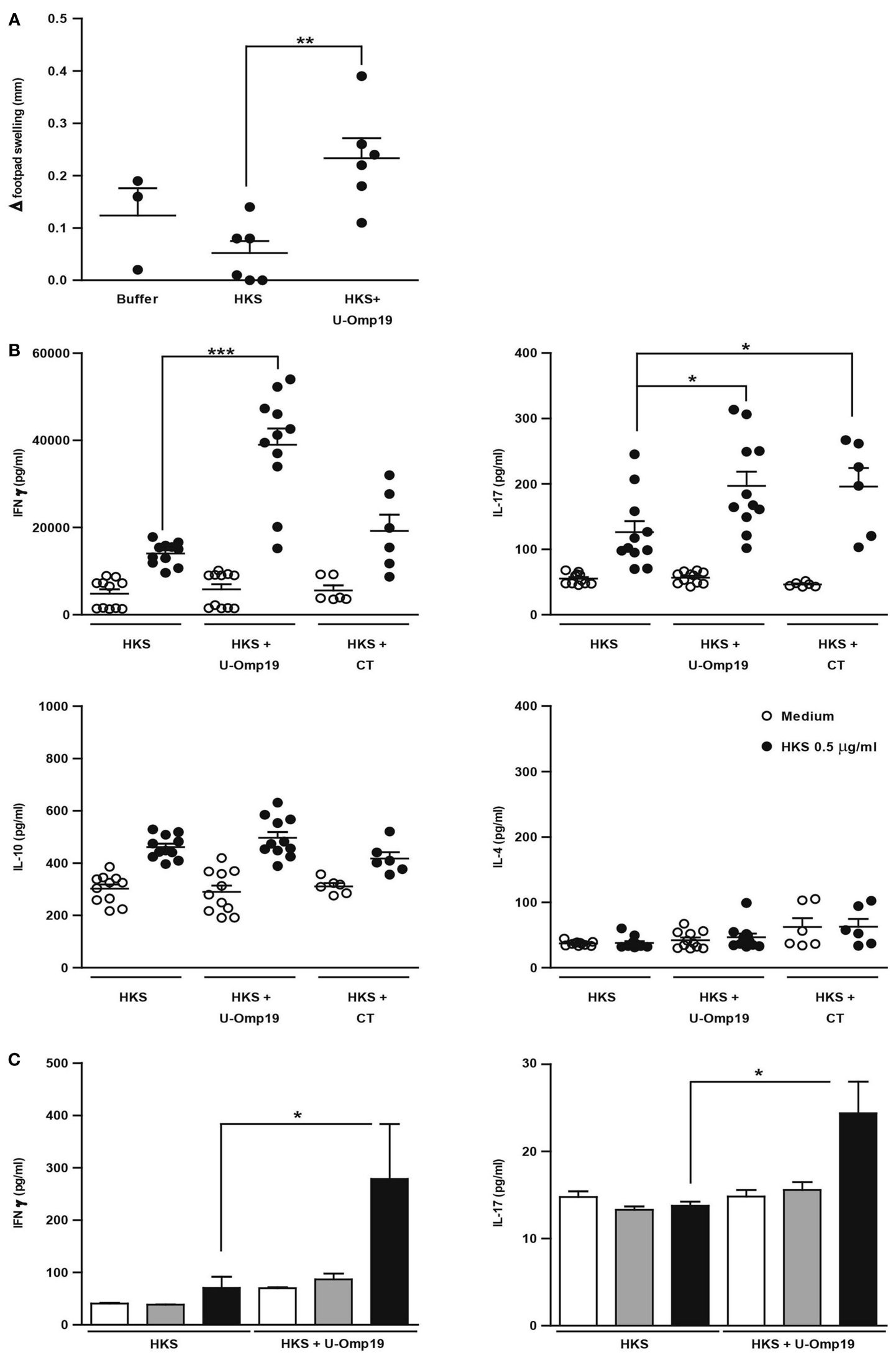

FIGURE 2 | Continued 
FIGURE 2 | Continued

U-Omp19 increases systemic and mucosal cellular immune response against HKS. (A) BALB/c mice were orally immunized on days 0 and 7 with buffer, HKS alone, or HKS + U-Omp19. Fourteen days post-last immunization, mice received an intradermal injection of HKS on one footpad and saline on the contralateral footpad. Swelling of each footpad was measured with a caliper $72 \mathrm{~h}$ later, and the difference between saline and HKS injected footpad was analyzed ${ }^{* *} p<0.01$; ANOVA plus Bonferroni's multiple comparisons test). (B) BALB/c mice were orally immunized on days 0 and 7 with HKS alone, HKS + U-Omp19, or HKS + CT. Spleens were obtained 14 days post-last immunization, and cells' suspensions were cultured in the presence of $0.5 \mu \mathrm{g} / \mathrm{ml}$ of $\mathrm{HKS}$ or complete medium. Supernatants were collected after 3 days, and cytokine production was determined by ELISA. Results from individual mice are shown as scatter dots ( ${ }^{*} p<0.05$, ${ }^{* * *} p<0.001$ vs HKS; Kruskall-Wallis plus Dunn's multiple comparisons test). (C) BALB/c mice were orally immunized on days 0 and 7 with HKS alone or HKS + U-Omp19. MLNs were obtained 14 days post-last immunization and, cells' suspensions were cultured in the presence of 0.5 or $10 \mu \mathrm{g} / \mathrm{ml}$ of HKS or complete medium. Supernatants were collected after 3 days, and cytokine production was determined by ELISA ( ${ }^{*} p<0.05$; Kruskal-Wallis plus Dunn's multiple comparisons test).

S. Typhimurium. Animals that received HKS + U-Omp19 presented a significant reduction in bacterial burden in spleen and liver compared to those that were administered with HKS alone (Figures 3A,B), indicating that addition of U-Omp19 to the HKS extract can increase protection against oral $S$. Typhimurium challenge. In contrast, animals immunized with HKS + CTB were not protected against infection. Importantly, U-Omp19 orally delivered alone did not induce reduction in bacterial burden when compared with buffer or HKS delivered mice (Figures 3C,D).

\section{U-Omp19 Maintains Its Adjuvant Capacity in a Chronic Outbred Mouse Model}

Though outbred animals may cause more variability in the experiments, they are more akin to the human population. Therefore, U-Omp19's adjuvant capacity in the CF-1 outbred mice strain was evaluated. Unlike BALB/c mice, CF-1 mice present a functional Nramp1 gene, they are notably less susceptible to Salmonella infection, and develop a chronic disease (30), which allows protection studies to be performed at longer time periods. Coadministration of U-Omp19 was able to enhance the protective capacity of HKS in this mouse strain, evidenced by a reduction in fecal shedding of bacteria and significantly lower CFU counts in liver compared to HKS alone. Immunization with HKS + CT had no effect on fecal shedding but produced a significant reduction in bacterial burden at the liver (Figure 4).

\section{U-Omp19 When Coadministered with Recombinant Salmonella Proteins Reduces Bacterial Burden after Salmonella Infection}

Subunit vaccination is an extremely safe method of immunization, it can be used on virtually everyone in need of vaccination regardless of health status and for that reason we sought to assess U-Omp19's adjuvant capability using recombinant Salmonella antigens. We chose two antigens that have previously been reported to reduce CFU count after infection: SseB, a part of the Salmonella Pathogenicity Island II, and Flagellin, which is the major structural protein of Salmonella flagellum (26-28). Preliminary studies showed that combination of these two antigens increases protective efficacy (S. J. McSorley, personal communication). Since U-Omp19 proved to have adjuvant properties when coadministered with recombinant protein antigens orally as well as systemic $(21,22)$, both routes were evaluated. In the
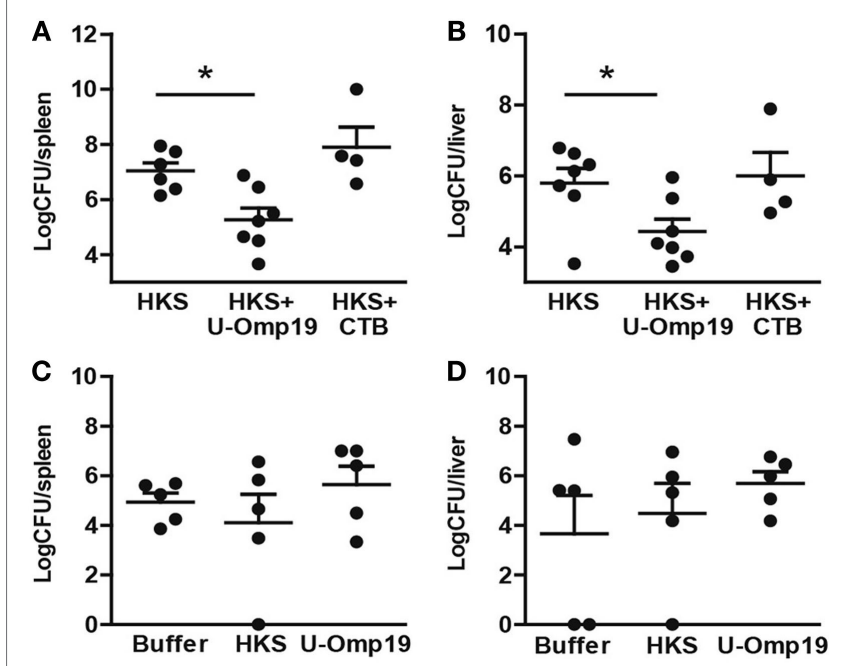

FIGURE 3 | Immunization with U-Omp19 + HKS reduces bacterial burden after Salmonella infection. (A,B) BALB/c mice were orally immunized on days 0 and 7 with HKS alone, HKS + U-Omp19, or HKS + CTB. Fourteen days post-last immunization, mice were orally infected with Salmonella Typhimurium. Six days postinfection mice were sacrificed, spleen (A) and liver (B) were harvested, and homogenates were plated on SS agar plates. (C,D) BALB/c mice were orally immunized on days 0 and 7 with HKS alone, buffer, or U-Omp19 alone. Fourteen days post-last immunization, mice were orally infected with $S$. Typhimurium. Six days postinfection mice were sacrificed, spleen (C) and liver (D) were harvested, and homogenates were plated on SS agar plates. CFU were counted after overnight incubation at $37^{\circ} \mathrm{C}$. Individual mice are shown as scatter dots $\left({ }^{*} p<0.05\right.$ vs HKS; ANOVA plus Bonferroni's multiple comparisons test).

systemic (i.v.) immunization protocol, U-Omp19 was as efficient as control adjuvant MPLA in reducing CFU counts after infection at spleen and liver of immunized mice compared to naive mice. Of note, in the oral immunization protocol, MPLA adjuvant was unable to induce protection against bacterial challenge, whereas mice immunized with U-Omp19 presented significantly lower bacterial burden at the liver (Figure 5).

\section{DISCUSSION}

Although most vaccines currently licensed are administered by systemic route, parenteral vaccination strategies fail to generate an adequate local mucosal immune response against still prevailing 

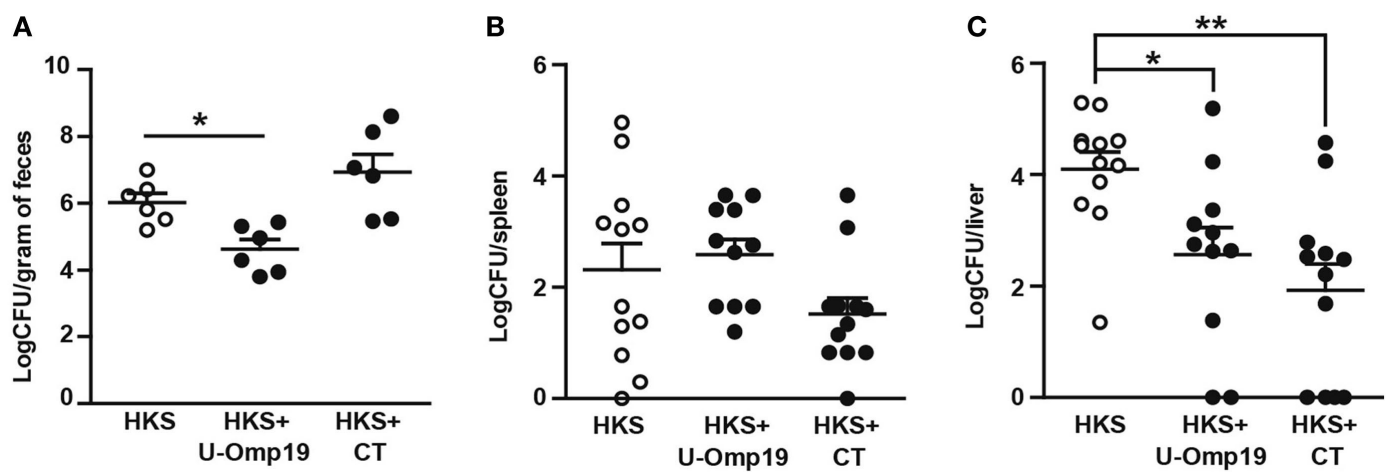

FIGURE 4 | Coadministration of U-Omp19 with HKS effectively reduces bacterial colonization in outbred mice after infection with Salmonella. CF-1 mice were orally immunized on days 0 and 7 with HKS alone, HKS + U-Omp19, or HKS + CT. Twenty-one days post-last immunization, mice were orally infected with Salmonella Typhimurium. (A) Four weeks postinfection, fecal pellets were suspended in PBS and plated on SS agar plates. For organ colonization experiments, 40 days postinfection mice were sacrificed, spleen (B) and liver (C) were harvested, and homogenates were plated on SS agar plates. CFU were counted after overnight incubation at $37^{\circ} \mathrm{C}$. Individual mice are shown as scatter dots $\left({ }^{*} p<0.05,{ }^{* *} p<0.01\right.$; Kruskal-Wallis plus Dunn's multiple comparisons test).

intestinal infections. On the contrary, oral immunization is capable of generating strong protective immunity at the intestinal mucosa as well as systemic (1-5). Particularly in developing countries, oral vaccination may offer a means to deal with safety concerns (associated with needle use) and the need for mass vaccination. However, in order to elicit an appropriate immune response, antigen digestion by proteases at the gastrointestinal tract must be avoided. Previous studies have shown that U-Omp19 has inhibitory activity over several proteases, in particular Pepsin present at the stomach (21). These results explain the observed protection of the HKS representative antigen flagellin from proteases present in a mouse stomach extract. As proteases at the stomach are constantly renewed, the effect of U-Omp 19 consists in a delay of the HKS extract degradation increasing its half-life. Using chicken OVA as a model antigen, it was demonstrated that this delay in antigen degradation increases the amount of antigen that reaches mucosal inductive sites (21). This may also be the case in the HKS model contributing to the induction of a stronger immune response against this antigen. Of note, study of other protease inhibitors has shown that the protease inhibitory activity by itself does not induce immune response against the coadministered antigen; it is the combination of U-Omp19's immunostimulatory properties together with a delay in antigen degradation that explain the adjuvant properties of U-Omp19 $(21,23)$.

The role of IgA in resistance against Salmonella infection is still controversial. Some state that antibodies against LPS, one of the components in the HKS extract, are associated to protection by blocking adhesion of bacteria to epithelial cells $(39,40)$, others declare that $\operatorname{IgA}$ is not required for protection since $\operatorname{IgA}$-deficient mice can be fully protected from infection (28), while others propose that the primary role of B cells in acquired immunity to Salmonella is via the development of protective $\mathrm{T}$ cell immunity (44), so the IgA increment would not be important per se but merely an immune correlate of B cell activation. In this work we demonstrated that after one oral boost, U-Omp19 can increase the amount of HKS-specific IgA in feces. Therefore, a role of this antibody increment in increased protection could be plausible. Also, coadministration of U-Omp19 did not induce a mucosal antibody response against itself, meaning that there would be no reduction of the adjuvant effect in a potential subsequent oral administration.

Cellular immune response has also been reported to be important for protection against Salmonella (41). Th1 cytokines and IFN- $\gamma$ in particular seem to be crucial during the initial stages of Salmonella growth since IFN- $\gamma^{-1-}$ mice develop high bacterial burden and succumb rapidly to infection (42). IL-17 as well has been implicated in the suppression of intestinal invasion by Salmonella (43). In previous work we described the role of U-Omp19 as self-adjuvant and adjuvant of model antigens (OVA) capable of shifting the immune response toward a Th1-Th17 profile $(20,21)$. In this work we provide additional evidence that supports the role of U-Omp19 as a Th1-Th17 oral adjuvant using antigens from a real pathogen. U-Omp19 was capable of inducing cell-mediated immune response in a DTH assay while increasing IFN- $\gamma$ and IL-17 production in splenocytes from immunized mice, which is consistent with a strong induction of a systemic immune response upon oral immunization with U-Omp19 as adjuvant. In these experiments, CT was able to induce an increase in IL-17 production, as previously reported in cell culture and mucosal immunization models for this adjuvant (45-47).

The mucosa is the front line of host defense against most pathogens. Therefore, induction of local immune response is essential to enhance the mucosal immune barrier that prevents infection by enteric pathogens (48). Our findings reveal that $\mathrm{U}-\mathrm{Omp} 19$ is capable of inducing increased levels of IFN- $\gamma$ and IL-17 at the MLNs, indicating the triggering of local immune response. These results are in accordance with previous findings using OVA as model antigen (21).

Moreover, the overall effect of coadministration of U-Omp19 with the antigens from HKS on antibody and cellular immune responses resulted in an increase in protection against challenge with a lethal dose of virulent $S$. Typhimurium in both inbred 

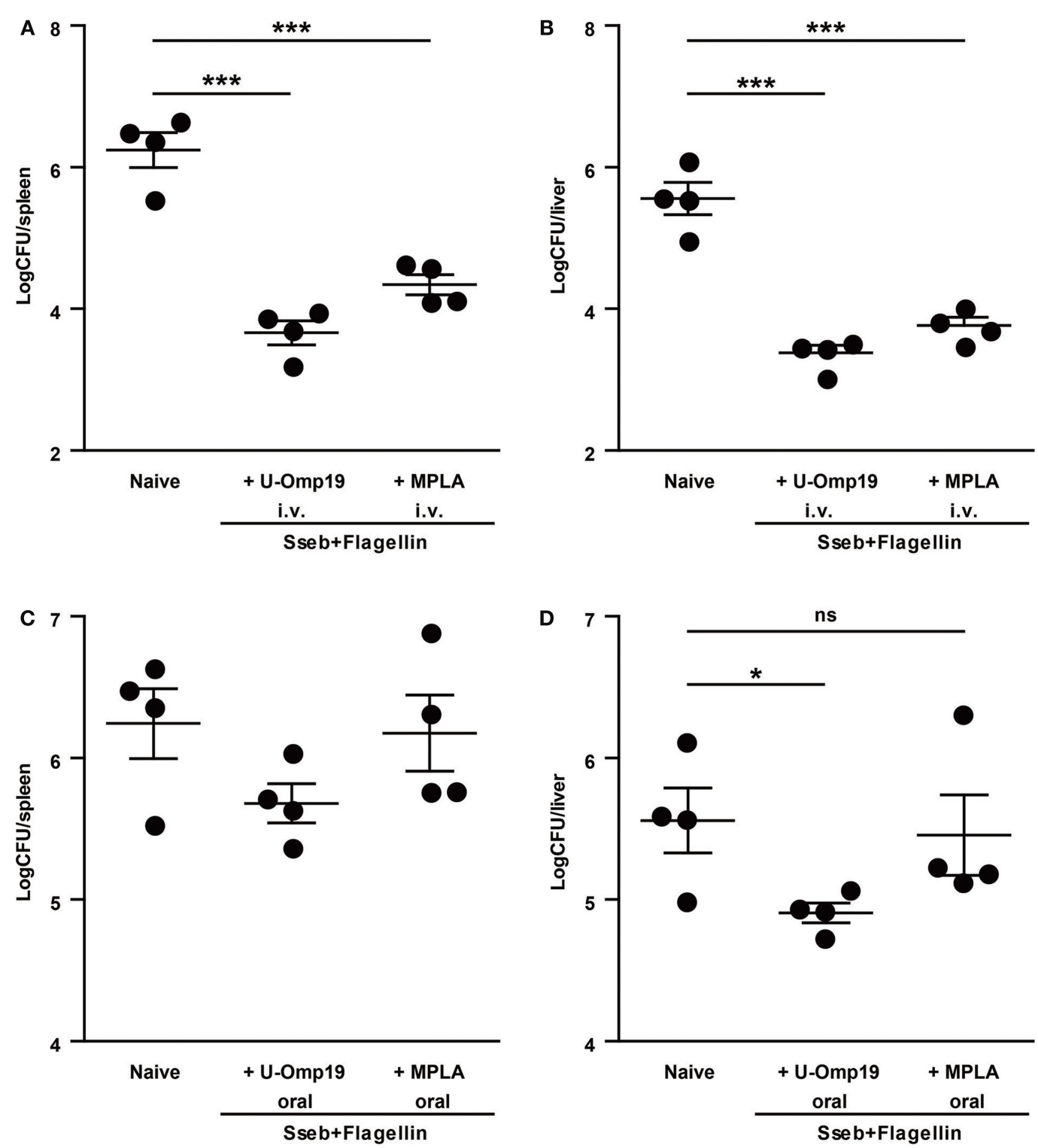

FIGURE 5 | U-Omp19 coadministered with recombinant Salmonella proteins reduces bacterial burden after Salmonella Typhimurium infection. BALB/c mice were immunized i.v. (A,B) or orally (C,D) on days 0 and 30 with buffer, SseB + Flagellin + U-Omp19, or SseB + Flagellin + MPLA. Thirty days post-last immunization, mice were i.v. (tail vein) infected with virulent $S$. Typhimurium. Four days later, spleen $\mathbf{( A , C )}$ and liver $(\mathbf{B}, \mathbf{D})$ were obtained, and bacterial load was determined by homogenization, serial dilution, and plating on MacConkey agar plates ( ${ }^{*} p<0.05,{ }^{\star \star \star} p<0.001$; ANOVA plus Bonferroni's multiple comparisons test).

BALB/c mice and outbred CF-1 mice. This proves that U-Omp19 is effective in different genetic backgrounds despite their intrinsic variability. Since U-Omp19 had no effect per se on protection against infection, the protective efficacy of the vaccine can only be attributed to U-Omp19's adjuvant effect when coadministered with the antigen. Overall the adjuvant capacity of U-Omp19 on inducing protection against Salmonella infection was superior to CTB in BALB/c and similar to CT in CF-1 mice. As CT cannot be administered orally to humans because of its enterotoxic effects, our results open new options in the progress of oral-killed vaccine formulations against Salmonella.
In addition to circumventing safety concerns, subunit vaccines present further advantages to inactivated vaccines: since their exact composition is known, they can be more reproducible, and also there is no need to manipulate the virulent microorganism during vaccine manufacture. For sure the future of vaccinology is headed toward subunit vaccine production. In this work we were able to demonstrate that $\mathrm{U}$-Omp19 is a suitable adjuvant in subunit vaccine formulations. Coadministration of U-Omp19 with recombinant Salmonella antigens intravenously was capable of reducing bacterial burden at spleen and liver after infection. Remarkably, U-Omp19 worked slightly better than MPLA, 
a well-known adjuvant approved by the U.S. Food and Drug Administration and used since 2009 in the Human Papillomavirus vaccine, Cervarix combined with aluminum hydroxide by the intramuscular route (49). Although i.v. immunization route is not applicable to human vaccination, our results show that this combination of antigens and U-Omp19 is capable of inducing protection against Salmonella infection by the i.v. route and encourages new studies combining these antigens and adjuvant with others and involving different means of vaccine delivery of relevance to human vaccination in the future.

Moreover, MPLA coadministered with the antigens by the oral route showed no signs of protection against infection, whereas U-Omp19 was able to induce a protective immune response evidenced by a significant reduction in bacterial burden at the liver of infected mice. These results open up a new range of possibilities for the rational design of oral subunit vaccines combining different recombinant antigens together with U-Omp19.

Full protection against infection is hard to achieve in this experimental model. However, the overall effect of coadministration of U-Omp19 with the antigens from HKS on antibody and cellular immune responses resulted in reduction of CFU counts after challenge with a lethal dose of virulent $S$. Typhimurium in both inbred BALB/c mice and outbred CF-1 mice. In the field of oral subunit vaccines, full protection is rarely achieved (30), and therefore, the reduction in bacterial load due to U-Omp19's adjuvant effect is a very encouraging scenario and calls for more studies to continue to optimize protective efficacy. Oral vaccine formulations containing newly discovered antigens and U-Omp19 potentially combined with other delivery systems and adjuvants, as is the case in many combined-adjuvant vaccines, could fully prevent Salmonella infection.

\section{REFERENCES}

1. Takahashi I, Nochi T, Yuki Y, Kiyono H. New horizon of mucosal immunity and vaccines. Curr Opin Immunol (2009) 21(3):352-8. doi:10.1016/ j.coi.2009.04.002

2. Gallichan WS, Rosenthal KL. Long-lived cytotoxic T lymphocyte memory in mucosal tissues after mucosal but not systemic immunization. J Exp Med (1996) 184(5):1879-90.

3. He XW, Wang F, Jiang L, Li J, Liu SK, Xiao ZY, et al. Induction of mucosal and systemic immune response by single-dose oral immunization with biodegradable microparticles containing DNA encoding HBsAg. J Gen Virol (2005) 86(Pt 3):601-10. doi:10.1099/vir.0.80575-0

4. Kim SY, Doh HJ, Ahn JS, Ha YJ, Jang MH, Chung SI, et al. Induction of mucosal and systemic immune response by oral immunization with $H$. pylori lysates encapsulated in poly(D,L-lactide-co-glycolide) microparticles. Vaccine (1999) 17(6):607-16.

5. Pavot V, Rochereau N, Genin C, Verrier B, Paul S. New insights in mucosal vaccine development. Vaccine (2012) 30(2):142-54. doi:10.1016/ j.vaccine.2011.11.003

6. Mestecky J, Russell MW, Elson CO. Perspectives on mucosal vaccines: is mucosal tolerance a barrier? J Immunol (2007) 179(9):5633-8. doi:10.4049/ jimmunol.179.9.5633

7. Holmgren J, Czerkinsky C. Mucosal immunity and vaccines. Nat Med (2005) 11(4 Suppl):S45-53. doi:10.1038/nm1213

8. Mowat AM. Anatomical basis of tolerance and immunity to intestinal antigens. Nat Rev Immunol (2003) 3(4):331-41. doi:10.1038/nri1057

9. Diamanti E, Ibrahimi B, Tafaj F, Mezini E, Dodbiba A, Dobi V, et al. Surveillance of suspected poliomyelitis in Albania, 1980-1995: suggestion of increased risk of vaccine associated poliomyelitis. Vaccine (1998) 16(9-10):940-8.
Inconclusion, thesefindingsindicate that oral coadministration of HKS with U-Omp19 can enhance both systemic and mucosal immunity against HKS and shift the immune response toward a Th1/Th17-like profile, and this effect translates into an increased level of protection against infection with $S$. Typhimurium.

\section{AUTHOR CONTRIBUTIONS}

GR and JC designed the experiments. GR lead all laboratory assays with assistance from MC, LB, AI, and LC. S-JL and LB performed subunit vaccine experiment. GR, JC, and KP performed all statistical analysis. SM and GB provided bacterial strains and, together with JC, contributed with their expertise on the subject. GR and JC interpreted all results and wrote the manuscript. All authors reviewed, commented, and approved the manuscript.

\section{FUNDING}

This work was supported by grants from the Bill and Melinda Gates Foundation through the Grand Challenges Explorations Initiative (OPP1060394 and OPP1119024); from the Agencia Nacional de Promoción Científica y Tecnológica (ANPCyTArgentina): PICT 2010 No 1163, PICT 2006 No 1670, ANPCyT/ CNPq PICT 2008 No 18 (to JC), and from NIAID (AI056172) to SM.

\section{SUPPLEMENTARY MATERIAL}

The Supplementary Material for this article can be found online at http://journal.frontiersin.org/article/10.3389/fimmu. 2017.00171/full\#supplementary-material.

10. Reed SG, Bertholet S, Coler RN, Friede M. New horizons in adjuvants for vaccine development. Trends Immunol (2009) 30(1):23-32. doi:10.1016/ j.it.2008.09.006

11. Coffman RL, Sher A, Seder RA. Vaccine adjuvants: putting innate immunity to work. Immunity (2010) 33(4):492-503. doi:10.1016/j.immuni.2010. 10.002

12. Freytag LC, Clements JD. Mucosal adjuvants. Vaccine (2005) 23(15):1804-13. doi:10.1016/j.vaccine.2004.11.010

13. Hill DR, Ford L, Lalloo DG. Oral cholera vaccines: use in clinical practice. Lancet Infect Dis (2006) 6(6):361-73. doi:10.1016/S1473-3099(06)70494-7

14. El-Kamary SS, Cohen MB, Bourgeois AL, Van De Verg L, Bauers N, Reymann $\mathrm{M}$, et al. Safety and immunogenicity of a single oral dose of recombinant double mutant heat-labile toxin derived from enterotoxigenic Escherichia coli. Clin Vaccine Immunol (2014) 20(11):1764-70. doi:10.1128/CVI.00075-14

15. Zhu Q, Talton J, Zhang G, Cunningham T, Wang Z, Waters RC, et al. Large intestine-targeted, nanoparticle-releasing oral vaccine to control genitorectal viral infection. Nat Med (2012) 18(8):1291-6. doi:10.1038/nm.2866

16. De Smet R, Demoor T, Verschuere S, Dullaers M, Ostroff GR, Leclercq G, et al. Beta-glucan microparticles are good candidates for mucosal antigen delivery in oral vaccination. J Control Release (2013) 172(3):671-8. doi:10.1016/ j.jconrel.2013.09.007

17. Kasturi SP, Skountzou I, Albrecht RA, Koutsonanos D, Hua T, Nakaya HI, et al. Programming the magnitude and persistence of antibody responses with innate immunity. Nature (2011) 470(7335):543-7. doi:10.1038/nature09737

18. Islam MA, Firdous J, Choi YJ, Yun CH, Cho CS. Design and application of chitosan microspheres as oral and nasal vaccine carriers: an updated review. Int J Nanomedicine (2012) 7:6077-93. doi:10.2147/IJN.S38330

19. Shima H, Watanabe T, Fukuda S, Fukuoka S, Ohara O, Ohno H. A novel mucosal vaccine targeting Peyer's patch $\mathrm{M}$ cells induces protective antigen-specific 
IgA responses. Int Immunol (2014) 26(11):619-25. doi:10.1093/intimm/ dxu061

20. Pasquevich KA, Ibanez AE, Coria LM, Garcia Samartino C, Estein SM, Zwerdling A, et al. An oral vaccine based on U-Omp19 induces protection against $B$. abortus mucosal challenge by inducing an adaptive IL-17 immune response in mice. PLoS One (2011) 6(1):e16203. doi:10.1371/journal. pone. 0016203

21. Ibanez AE, Coria LM, Carabajal MV, Delpino MV, Risso GS, Cobiello PG, et al. A bacterial protease inhibitor protects antigens delivered in oral vaccines from digestion while triggering specific mucosal immune responses. J Control Release (2015) 220(Pt A):18-28. doi:10.1016/j.jconrel.2015.10.011

22. Coria LM, Ibanez AE, Pasquevich KA, Cobiello PL, Frank FM, Giambartolomei $\mathrm{GH}$, et al. Brucella abortus Omp19 recombinant protein subcutaneously co-delivered with an antigen enhances antigen-specific T helper 1 memory responses and induces protection against parasite challenge. Vaccine (2016) 34(4):430-7. doi:10.1016/j.vaccine.2015.12.012

23. Coria LM, Ibanez AE, Tkach M, Sabbione F, Bruno L, Carabajal MV, et al. A Brucella spp. protease inhibitor limits antigen lysosomal proteolysis, increases cross-presentation, and enhances CD8+ T cell responses. J Immunol (2016) 196(10):4014-29. doi:10.4049/jimmunol.1501188

24. Jones BD, Falkow S. Salmonellosis: host immune responses and bacterial virulence determinants. Annu Rev Immunol (1996) 14:533-61.

25. Song J, Willinger T, Rongvaux A, Eynon EE, Stevens S, Manz MG, et al. A mouse model for the human pathogen Salmonella typhi. Cell Host Microbe (2010) 8(4):369-76. doi:10.1016/j.chom.2010.09.003

26. Strindelius L, Degling Wikingsson L, Sjoholm I. Extracellular antigens from Salmonella enteritidis induce effective immune response in mice after oral vaccination. Infect Immun (2002) 70(3):1434-42. doi:10.1128/ IAI.70.3.1434-1442.2002

27. McSorley SJ, Cookson BT, Jenkins MK. Characterization of CD4+ T cell responses during natural infection with Salmonella typhimurium. J Immunol (2000) 164(2):986-93. doi:10.4049/jimmunol.164.2.986

28. Lee SJ, Liang L, Juarez S, Nanton MR, Gondwe EN, Msefula CL, et al. Identification of a common immune signature in murine and human systemic Salmonellosis. Proc Natl Acad Sci U S A (2012) 109(13):4998-5003. doi:10.1073/pnas.1111413109

29. Moon JJ, McSorley SJ. Tracking the dynamics of Salmonella specific T cell responses. Curr Top Microbiol Immunol (2009) 334:179-98. doi:10.1007/978-3-540-93864-4_8

30. Simon R, Tennant SM, Galen JE, Levine MM. Mouse models to assess the efficacy of non-typhoidal Salmonella vaccines: revisiting the role of host innate susceptibility and routes of challenge. Vaccine (2011) 29(32):5094-106. doi:10.1016/j.vaccine.2011.05.022

31. Monack DM, Bouley DM, Falkow S. Salmonella typhimurium persists within macrophages in the mesenteric lymph nodes of chronically infected Nramp1+/+ mice and can be reactivated by IFNgamma neutralization. J Exp Med (2004) 199(2):231-41. doi:10.1084/jem.20031319

32. Santos RL, Zhang S, Tsolis RM, Kingsley RA, Adams LG, Baumler AJ. Animal models of Salmonella infections: enteritis versus typhoid fever. Microbes Infect (2001) 3(14-15):1335-44. doi:10.1016/S1286-4579(01)01495-2

33. Pasquevich KA, Estein SM, Samartino CG, Zwerdling A, Coria LM, Barrionuevo P, et al. Immunization with recombinant Brucella species outer membrane protein Omp16 or Omp19 in adjuvant induces specific CD4+ and CD8+ T cells as well as systemic and oral protection against Brucella abortus infection. Infect Immun (2009) 77(1):436-45. doi:10.1128/IAI. 01151-08

34. Atif SM, Lee SJ, Li LX, Uematsu S, Akira S, Gorjestani S, et al. Rapid CD4+ T-cell responses to bacterial flagellin require dendritic cell expression of Syk and CARD9. Eur J Immunol (2015) 45(2):513-24. doi:10.1002/eji.201444744

35. Lycke N, Holmgren J. Strong adjuvant properties of cholera toxin on gut mucosal immune responses to orally presented antigens. Immunology (1986) 59(2):301-8.

36. Vajdy M, Lycke NY. Cholera toxin adjuvant promotes long-term immunological memory in the gut mucosa to unrelated immunogens after oral immunization. Immunology (1992) 75(3):488-92.

37. Jackson RJ, Fujihashi K, Xu-Amano J, Kiyono H, Elson CO, McGhee JR. Optimizing oral vaccines: induction of systemic and mucosal B-cell and antibody responses to tetanus toxoid by use of cholera toxin as an adjuvant. Infect Immun (1993) 61(10):4272-9.

38. Lee A, Chen M. Successful immunization against gastric infection with Helicobacter species: use of a cholera toxin B-subunit-whole-cell vaccine. Infect Immun (1994) 62(8):3594-7.

39. Forbes SJ, Eschmann M, Mantis NJ. Inhibition of Salmonella enterica serovar Typhimurium motility and entry into epithelial cells by a protective antilipopolysaccharide monoclonal immunoglobulin A antibody. Infect Immun (2008) 76(9):4137-44. doi:10.1128/IAI.00416-08

40. Mantis NJ, Forbes SJ, Easchmann M, Bumpus T. Protective anti-lipopolysaccharide monoclonal IgA antibodies interferes with Salmonella motility and invasion of epithelial cells, independent of bacterial agglutination. FASEB J (2008) 22(Suppl 852.19). Available from: http://www.fasebj.org/cgi/content/ meeting_abstract/22/1_MeetingAbstracts/852.19

41. Kupz A, Bedoui S, Strugnell RA. Cellular requirements for systemic control of Salmonella Typhimurium infections in mice. Infect Immun (2014) 82(12):4997-5004. doi:10.1128/IAI.02192-14

42. Bao S, Beagley KW, France MP, Shen J, Husband AJ. Interferon-gamma plays a critical role in intestinal immunity against Salmonella typhimurium infection. Immunology (2000) 99(3):464-72. doi:10.1046/j.1365-2567.2000.00955.x

43. Mayuzumi H, Inagaki-Ohara K, Uyttenhove C, Okamoto Y, Matsuzaki G. Interleukin-17A is required to suppress invasion of Salmonella enterica serovar Typhimurium to enteric mucosa. Immunology (2010) 131(3):377-85. doi:10.1111/j.1365-2567.2010.03310.x

44. Nanton MR, Way SS, Shlomchik MJ, McSorley SJ. Cutting edge: B cells are essential for protective immunity against Salmonella independent of antibody secretion. J Immunol (2012) 189(12):5503-7. doi:10.4049/jimmunol.1201413

45. Lee JB, Jang JE, Song MK, Chang J. Intranasal delivery of cholera toxin induces th17-dominated T-cell response to bystander antigens. PLoS One (2009) 4(4):e5190. doi:10.1371/journal.pone.0005190

46. Tsai HC, Wu R. Cholera toxin directly enhances IL-17A production from human CD4+ T cells. J Immunol (2013) 191(8):4095-102. doi:10.4049/ jimmunol.1301079

47. Griffiths KL, Stylianou E, Poyntz HC, Betts GJ, Fletcher HA, McShane H. Cholera toxin enhances vaccine-induced protection against Mycobacterium tuberculosis challenge in mice. PLoS One (2013) 8(10):e78312. doi:10.1371/ journal.pone.0078312

48. Hammerschmidt SI, Friedrichsen M, Boelter J, Lyszkiewicz M, Kremmer $\mathrm{E}$, Pabst $\mathrm{O}$, et al. Retinoic acid induces homing of protective $\mathrm{T}$ and $\mathrm{B}$ cells to the gut after subcutaneous immunization in mice. JClin Invest (2011) 121(8):3051-61. doi:10.1172/JCI44262

49. Harper DM, Franco EL, Wheeler CM, Moscicki AB, Romanowski B, Roteli-Martins CM, et al. Sustained efficacy up to 4.5 years of a bivalent L1 virus-like particle vaccine against human papillomavirus types 16 and 18: follow-up from a randomised control trial. Lancet (2006) 367(9518):1247-55. doi:10.1016/S0140-6736(06)68439-0

Conflict of Interest Statement: The authors declare that the research was conducted in the absence of any commercial or financial relationships that could be construed as a potential conflict of interest. LC, AI, KP, and JC are inventors on a patent related to U-Omp19. This patent, presented by the authors' National Research Council, "Adjuvant for vaccines, vaccines that comprise it and uses," presentation P 20090104015, was filed on October 19, 2009 in the National Institute of Intellectual Property, Argentina. This patent was also filed on October 18, 2010 in the European Patent Office, Spain PCT/ES2010/070667. EP2491946B1, CN102711815B, and DK2491946T3 granted. The filing of the patent did not have any role in experimental design, data collection and analysis, decision to publish, or preparation of this manuscript.

Copyright $\odot 2017$ Risso, Carabajal, Bruno, Ibañez, Coria, Pasquevich, Lee, McSorley, Briones and Cassataro. This is an open-access article distributed under the terms of the Creative Commons Attribution License (CC BY). The use, distribution or reproduction in other forums is permitted, provided the original author(s) or licensor are credited and that the original publication in this journal is cited, in accordance with accepted academic practice. No use, distribution or reproduction is permitted which does not comply with these terms. 